\title{
Prototype Design of a Remote Medical Monitoring System Based on the Internet of Things
}

\author{
http://dx.doi.org/10.3991/ijoe.v12i1.5227 \\ XiuMing ZOU \\ Huaiyin Normal University, Huaian, Jiangsu, China
}

\begin{abstract}
Because remote medical monitoring systems are highly distributed, limited to node energy and have a large quantity of process and transmission information, this paper proposes a kind of remote medical monitoring system based on the technology of the Internet of things combined with a mobile agent. Ten patients $(5$ male and 5 female, ages 18 69 with an average age of 41.8) were given a clinical test with this system. The paper shows the architectural model of the system consists of wireless sensor nodes, relay nodes, a remote monitoring center, etc. To collect the patients' physiological data, the remote monitoring center in the hospital issues a mobile agent and runs it in a wireless sensor node. After processing the mobile agent, the center sends the data to the relay node and then back to the hospital's remote monitoring center through the Internet of Things to do comprehensive diagnosis and make decisions. The project has completed the software and hardware design and debugging of each module of the system. The results show that the system is able to monitor the health status of patients who live in different communities in the long term and in time. Furthermore, the system can achieve optimal utilization of medical resources.
\end{abstract}

Index Terms-Mobile Agent; Remote telemedicine monitoring system; Wireless sensor network

\section{INTRODUCTION}

With China facing an aging society, the proportion of the elderly population is increasing. Therefore, solving how to monitor elderly men's long-term chronic disease, how to provide a safe medical monitoring environment for the elderly men, and how to ensure the security of the elderly men's health and life have become important social problems [1]. Also due to the accelerating pace of life, hypertension, hyperlipidemia, and sudden cardiovascular and cerebrovascular diseases have been threatening the health of young people. The trend of the medical models is focusing more in the direction of grassroots communities and individual participation. Personal health monitoring and diagnosis by family can find illness quickly and help doctors take immediate emergency measures to reduce damage to the human body [2-3].

In recent years, the study of remote medical monitoring systems based on network technology has became a hot topic [4-6]. Bhatia et al. proposed a kind of remote consultation and diagnosis system in which patients can provide the remote medical server with illness information via the Internet, and then medical staff can confirm the results or give suggestions. The system consists of the monitoring center, remote monitoring equipment and the communica- tion network for contacting the center through the equipment. Thus, this system is a remote diagnosis and nursing consultation system, but it lacks certain intelligence [7], so it needs medical staff to monitor patients' condition 24 hour a day and at the same time review and explain patients' information, perform diagnostic tasks, and confirm treatment plans. When patients are involved in outdoor activities, monitoring equipment and lead cables can interfere with the patients' activity, so the application of this method is restricted to certain degree. Vaidehi et al. used wireless sensor network technology to design an elderly guardianship system based on a wireless sensor network and RFID technology. This system could be simply installed and could easily monitor, diagnose, and orient the elderly health status information. The C/S network architecture transmits a large amount of redundant information between nodes and will deplete the nodes' energy prematurely and make nodes fail. The worst situation will affect the network performance of the whole system [8], reducing the life of the system. Cai proposed an intelligent monitoring system development plan in the Internet of Things to meet the demand for intelligent monitoring terminal communication and sensing and presented a design for smart intelligent monitoring systems. But this system is still in the laboratory research stage, so if it faces monitoring objects from a large area, its restriction of communication bandwidth and node energy will constrain the application of the system [9].

This paper combines the Internet of Things technology with mobile agent technology to design a remote medical monitoring system, and ultra-low power consumption and small size sensor nodes can be designed suitable for human body health checks, so patients can easily install or carry and effectively extend the service life of node; multi-object route algorithms between nodes based on mobile agent can be studied to efficiently process and transmit data. Radio frequency identification (RFID) technology can be applied to remote medical monitoring systems. Every patient can wear wristbands embedded with RFID to store the information, and then medical staff can quickly identify patients with communication difficulties and come up with effective treatment as soon as possible. The system allows the mobile subject to perform part of the function of the medical staff, collecting the patient's vital signs data in different locations and spontaneously informing irregular status to a related physician in time to realize health services in the community and family [9-10]. 


\section{MATERIALS AND METHODS}

A. Architectural design of remote medical monitoring system based on the Internet of Things and mobile agent technology

The Internet of Things means that in accordance with the network transmission protocol, it can connect any item with the internet for information exchange and communication through sensing equipment like RFID, sensors, a global positioning system (GPS), and a scanner and realize a comprehensive network to recognize between objects and objects and people and objects. The generation and development of internet technology promote the intelligentization and informatization of medical technology. Intelligent remote monitoring based on the technology of the Internet of Things can make the collection and transmission of patients' physiological parameters more convenient and faster. Patients can communicate with medical experts any time via personal computers, personal digital assistants (PDAs), telephone, email, etc., and therefore, in home professional medical health service can be realized with the best medical effect, the lowest medical cost, and the shortest medical treatment time.

According to the technical requirements and development trend of existing remote monitoring systems, this paper proposes a kind of remote medical monitoring system that has a three-layer architecture: -- "home monitoring nodes, community monitoring center, and hospital monitoring center" with the Internet of Things technology as the core. Therefore, the system can realize health management, health monitoring, remote medical help, and so on, and these functions can interact and interlock, so the system can guarantee the monitoring of individuals' health. The designed architecture of remote medical monitoring system based on the Internet of Things technology is shown in Figure 1.

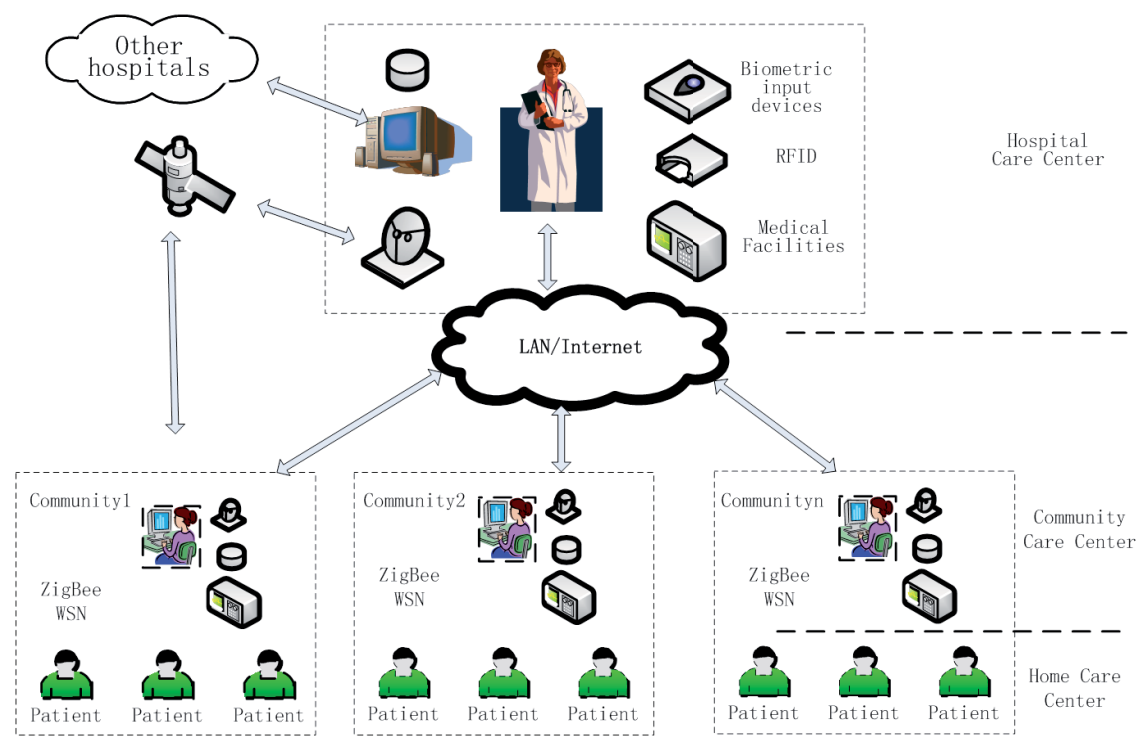

Figure 1. Three-tier architecture of remote medical monitoring system based on Internet of Things

In the family monitoring nodes level, the system collects human vital signs, such as temperature, electrocardiogram (ECG), electroencephalograph (EEG), pulse, blood oxygen, blood pressure and so on through the integration of wireless sensor nodes worn by patients, and then this information, which has been through the nodes' wireless transceiver modules, will be sent to the community monitoring center by the self-organizing and multi-hop wireless network.

In the community care center, signals of human body life signs, which are sent by the patients wearing the wireless sensor nodes, can be received by the relay nodes and preliminarily processed. When the center finds an abnormal signal, it will issue instructions to the patient that further checking is immediately needed in the community care center, and the community doctors can identify general illness. For complex or critical illnesses, the community relay station will transmit the patients' vital sign parameters to the large-scale hospital monitoring center through a local area network (LAN).

The hospital remote monitoring center sets a central database that preserves the medical institution's basic personal data, medical behavior data, decision-making data, etc. The center sets up the Internet of Things communication system through the existing Internet, satellites and $3 \mathrm{G} / 4 \mathrm{G}$ networks, and automatically receives, analyzes, processes, and diagnoses patients' physiological characteristics data sent by the community care center, and then determines a result. At the same time, the raw data transmitted by the intelligent terminal can also be stored for further diagnosis and decisions. For hospitalized critical patients, the patient's identity can be quickly identified by the RFID, the patients' past medical history can be read and analyzed, and a quick and accurate treatment can be given.

As shown in Figure 1, the monitoring system is composed of several community care centers. In each monitoring center, the doctor on duty needs to monitor patients in different families in the community on different physiological parameters, such as blood pressure, ECG, pulse, etc. Traditional monitoring methods need to use fixed medical monitoring equipment, and the large number of connection cables allows the sensor probes to connect patients and medical equipment to realize signal transmission; therefore, sophisticated equipment and numerous signal connections not only bring psychological pressure for patients and influence the effect of treatment, but they also create inconvenience for patients and doctors and restrict the freedom of patients' activities. 
The wireless sensor network can be used to monitor patients' illness, which could effectively reduce the number of connection cables and make the installation and debugging of the system more convenient. Thus, the difficulties faced by traditional monitoring methods can be effectively solved. This project used a ZigBee wireless sensor network to design the wireless medical community monitoring system based on $\mathrm{C} / \mathrm{S}$ structure as shown in
Figure 2. The sensor nodes that patients wear are responsible for collecting patients' human physiological data, preliminarily processing signals, and then sending data through the relay station to the community care center to make comprehensive diagnoses and decisions. This system allows patients more freedom, and they are exempted from the hustle and bustle of travelling between the hospital and home [11-13].

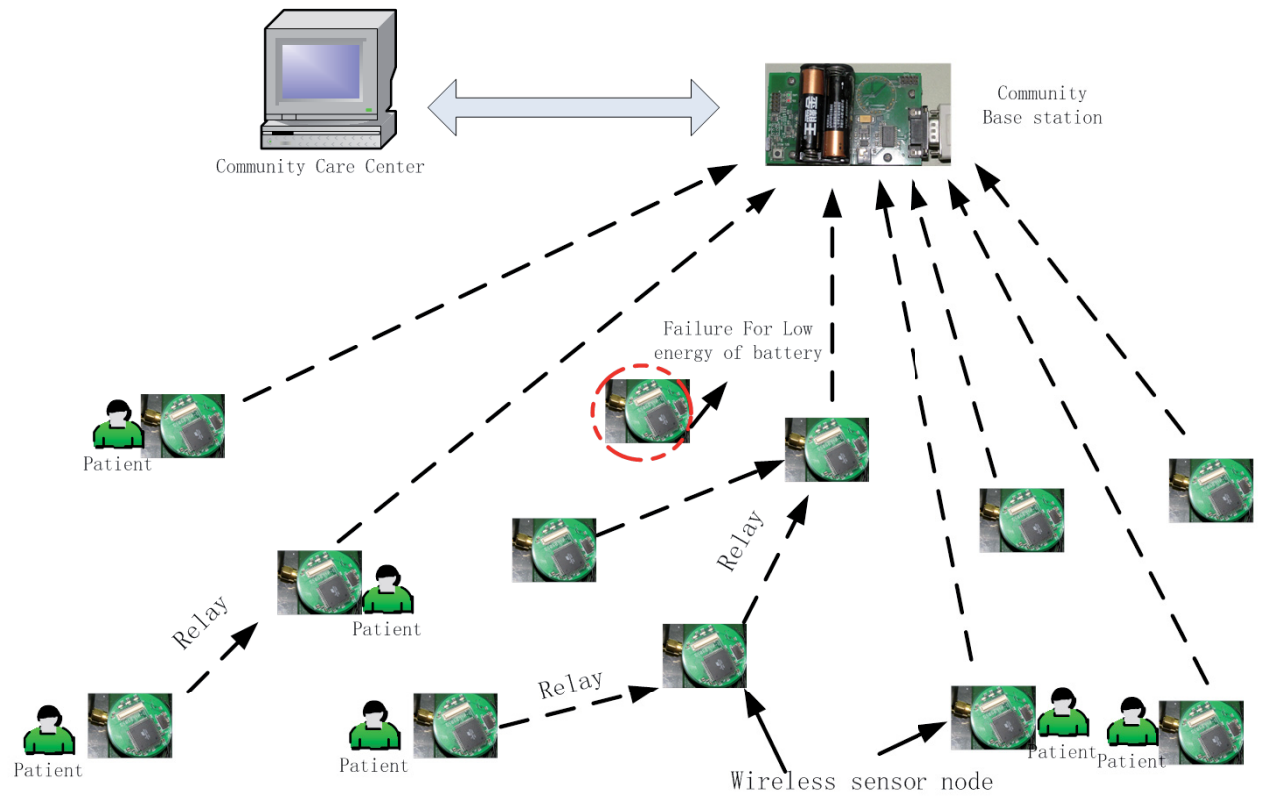

Figure 2. Community medical monitoring system model based on $\mathrm{C} / \mathrm{S}$ structure

A remote medical monitoring system based on a wireless sensor network can effectively collect, transmit and process health care information, clinical information and medical records long distance; thus, it is an information exchange platform for medical professionals and patients. But these client systems are mobile wireless sensor nodes, so they are low bandwidth and unstable in connecting communication environments, and their servers are distributed and isomerous. Therefore, there are some difficulties in exchanging information. Mobile agent technology provides a powerful and efficient mechanism for application and development in distributed heterogeneous environments, and the mechanism can effectively solve these problems. In a remote medical monitoring system based on wireless sensor networks, introducing mobile agent technology can effectively overcome the limitations of client devices, solve the difficulties of information transmission among heterogeneous devices and automatically process a large amount of patient health status data. As a result, a lot of valuable medical information can be saved for medical personnel [14-16].

As shown in Figure 2, the system is open and heterogeneous. With the monitoring target area and the number of sensor nodes increased, the monitoring system has large distribution characteristics, and the transmission and processing of data increase. At the same time, the consumption of the sensor nodes' energy also increases sharply and unevenly, especially nodes near the base station node are depleted of energy and prematurely fail from the frequency of the relay state. However, based on the system shown in Figure 2, mobile agent technology can be used to optimize the software structure of a monitoring system. A mobile agent provides unique distributed architecture. Thus, the community care center can create multiple mobile agents, move them to different nodes, and run them at the same time, which can distribute the load of a single node to multiple nodes in the network. Therefore, the small system has the ability to deal with large-scale and complicated problems. The mobile agent structure and its migration process in a heterogeneous environment are shown in Figure 3.

The path of mobile agent migration has a great influence on system performance. This paper, based on the characteristics of a community medical monitoring system, puts forward a routing algorithm based on multiple target fusion (MOF) algorithms to plan mobile agent routes [17]. First, the data packet and data tables need defining to gain the basic information of the target node, whose energy level is more than the setting threshold value and then considers the condition of frequent sensor node failure. The optimal path from these nodes to the processing nodes can be found. A message can be divided into three categories:

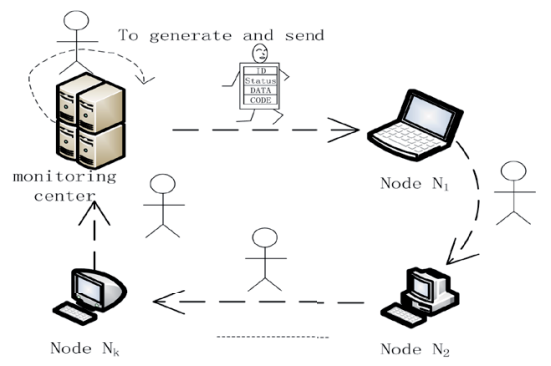

Figure 3. Mobile Agent structure and migration in heterogeneous environment 
Node packets (NIP): generated by each node in the construction of local network topology and broadcast to neighbor nodes - defined as 4 groups (TYPE, IDN, POS, EN). Among them, TYPE means the type of bag; IDN means the node's ID number (ID); POS means the node's location; and EN means the node's residual energy. Ask package (ASKP): generated by processing nodes and limited by flooding in the network. Three groups are defined (TYPE, IDP, ETH). Among them, TYPE means the type of bag; IDP means the processing nodes' ID; and ETH means the minimum residual node energy for processing a task (residual energy threshold). Answer packet (ANSP): defined as 4 groups (TYPE, IDS, IDP, EN). Among them, TYPE means the type of bag; IDS means the reply nodes' ID; IDP means the processing nodes' ID; and EN means the reply nodes' residual energy.

In an MOF algorithm, the mobile agent needs to not only consider the residual energy of each sensor node on the moving path, but also move on the optimal path. The mobile agent must interact with sensor nodes in the network environment and query the current residual energy, location information, and information ability of each sensor node to realize the balance of each node consumption and prevent an individual premature sensor due to the heavy task, resulting in a decline in network performance and failure. The algorithm is described as the following:

Step 1: At the time of $t=0$, the sensor node that first perceives the monitoring targets will be selected as the relay node to create the mobile agent (MA). This sensor node will estimate the target location at the point of $t=0$ according to the messages transmitted by neighboring nodes and the target tracking algorithm, and then the current information on the sensor nodes can be calculated. The initialized information will carry the quantity of carry $=1$; from the target's estimated position, MA migration energy consumption information and a node residual energy cost model at $\mathrm{t}=0$, the next hop node can be selected.

Step 2: At the time of $t$, the mobile agent has reached the sensor node $\mathrm{k}$. This sensor node estimates the target's current position according to the target tracking algorithm; the information achievement ik on the sensor node $\mathrm{k}$ can be calculated and the updated information carrying amount is carry $=$ carry $+\mathrm{ik}$, IF carry $>$ desire (information carrying amount of mobile agent device is in advance), Then it goes to step 3; otherwise, it will continue to determine the next-hop sensor nodes through the target's estimated location, the calculation of energy consumption at the time of $t$, update the time of $t=t+1$, and then repeat step 2.

Step 3: Return to the relay node and create a mobile agent. At the same time, assign an initial value that carries the desired information amount. The information collected by the mobile agent in each sensor node will be saved in a carry variable. After updating the carry in every migration, the two values will be compared. If the value of carry has reached the required information carrying capacity, the mobile agent can return to the relay node for further processing according to the original path or migration of the mobile agent will continue and gather more information.

B. Design of remote medical monitoring system

Based on the above analysis, this project designed remote medical monitoring system architecture based on the Internet of Things and mobile agent technology, as shown in Figure 4. The system includes wireless sensor nodes, relay nodes, a community care center, a hospital monitoring center, etc. The hospital monitoring center or community care sends the mobile agent to the target node according to the need to perform corresponding monitoring tasks. When the mobile agent arrives at the target node, it starts and collects patients' blood pressure, pulse, ECG and other physiological parameters, and then processes the desired results and carries the results to the hospital monitoring center or community care center through relay nodes, and eventually the monitoring center can make a comprehensive diagnosis and decision.

The role of the sensor nodes is that patients' health status can be monitored; the health status here includes the heart and brain health status, the characteristic parameters,

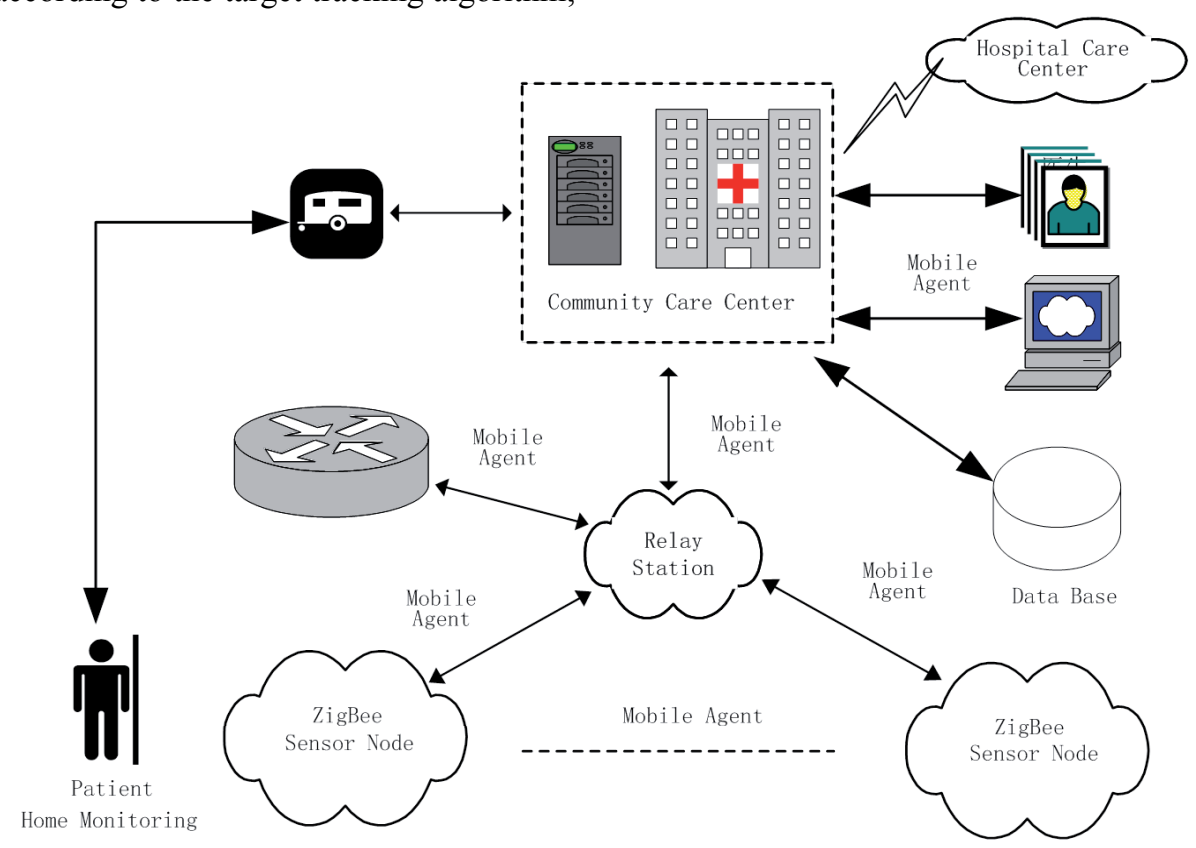

Figure 4. Remote medical monitoring system structure based on Internet of Things and mobile Agent technology 
such as blood pressure, ECG, EEG, body temperature, pulse and so on. The relay node is responsible for receiving and processing the patient's health information transmitted by a nearby wireless sensor node, and for then sending the preliminary results to the community care center via the Internet or LAN. Relay nodes must possess a higher ability to store energy than sensor nodes. A community care center can process, diagnose, and interpret the data from the sensor nodes. For critically ill patients, it will send information to the hospital monitoring center through the Internet of Things system to order pertinent treatment.

The remote medical monitoring requirements are small wireless sensor nodes with limited power supply capacity [18-19] and a set of signal pretreatment circuits with low consumption. High gain and great ability to de-noise are needed to pretreat the front end amplifying and filtering of the ECG and EEG signals, but the suitable wireless sensor network nodes that match the human body's physiological parameters are less. Therefore, the point of developing the system is to design low consumption wireless sensor nodes and relay nodes that can meet the requirements for remote medical monitoring. To optimize the design of the wireless sensor nodes and further reduce the power consumption of the node, single-chip microcomputer control modules for wireless remote medical monitoring sensor nodes and base stations will use a 16 bit low power consumption microprocessor MSP430F169 from the TI Company. The block diagram of the wireless sensor nodes and relay nodes facing the remote medical monitoring are shown in Figure 5. The system hardware uses modularity and an independent hierarchy for remote medical monitoring wireless sensor nodes; they mainly consist of front-end sensor signal collection and processing, an RFID module, a control module for a singlechip microcomputer, a wireless radio frequency transceiver, the power supply, etc., and in the module of the front-end sensor signal collection, it includes an ECG module, pulse module, temperature module, EEG module, etc. The wireless transceiver module uses CC2420 as the core chip; it works in an ISM frequency band, and the communication protocol adopts a ZigBee protocol. The communication frequency is $2.4 \mathrm{GHz}$, and the bandwidth is $8 \mathrm{MHZ}$. The PCB can be used to make a micro-strip line as a signal coupling antenna, and then the matching circuit can be designed. The circuit can send and receive wireless signals.

The software of a remote medical monitoring system based on the Internet of Things includes monitoring center software, monitoring client end software and sensor node software. The monitoring center software uses Lab View and Java language to develop and complete the initialization of the module, to assign and coordinate each mobile agent module to use system resources, to make comprehensive decisions for the data monitored by the wireless sensor nodes, and to warn and send data to relevant doctors according to the results of the monitoring. It provides the community doctors with a convenient monitoring platform, ensures that abnormal patients can obtain guidance as soon as possible, and provides preliminary treatment opinions and emergency nursing work. According to the function modules, the structure of the monitoring system software can be divided into subjects of communication, subjects of user interface, subjects of resources, subjects of doctor, subjects of diagnosis, subjects of data services, subjects of related mobile, etc. Figure 6 shows the mobile body architecture of the platform. The subjects of the user interface are the bridges for hosts and users. The UIA acts as an interpreter between heterogeneous subjects and applications; it accepts the requests of UIA applications and awakens the internal services. Therefore, the UIA is an access control mechanism and can identify the users before starting the service. The UIA is responsible for having a final statement about the mobile subject's results or diagnosis subject results before the data are transferred to the application layer.

The coordination of the main resources is accessed from mobile subjects to hosts and provides expert advice or local service. The main resources play an important

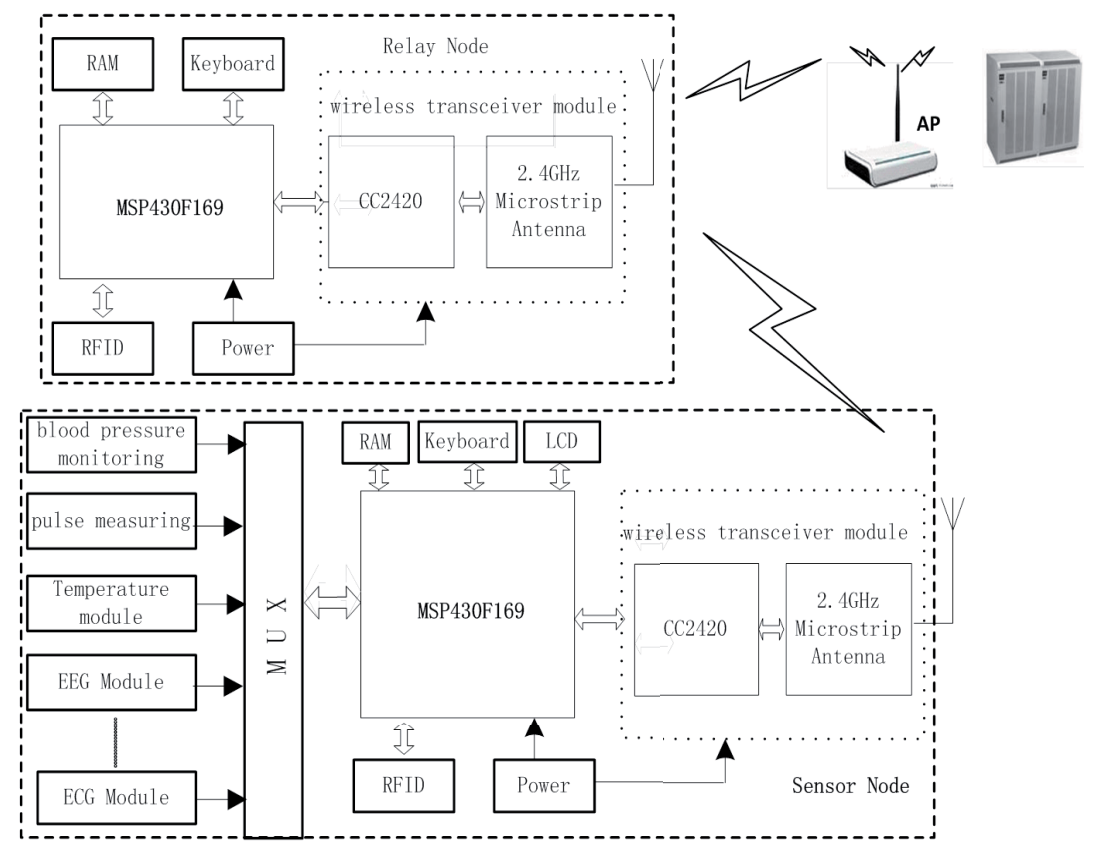

Figure 5. Block diagram of wireless sensor nodes and relay nodes facing medical care 
role in the dynamic connection with the host resources. Through the main resources, the mobile body can access the resources of the host system. The doctor subject is used as a moving subject by medical personnel, and it may help medical personnel perform their tasks and monitorpatients' conditions. Medical staff can use their own mobile devices to inspire their doctor subjects and then send them to the specific subject platform. The doctor subject will continue to perform assigned tasks according to the characteristics of given clinical cases. The doctor subject is dedicated to collecting data and then carrying the collected data back to the host server.

The doctor terminals can be sent to several terminals according to different departments; the display module is a free mobile tablet. Because the tablet has the advantage of good maneuverability and freedom, it can let users walk freely in the hospital, help doctors process and arrange things conveniently, help doctors determine the etiology of patients accurately, and provide the basis for further arrangements.

The diagnosis body could be considered as a data analysis engine. It can analyze data collected from the vital signs monitoring sensor nodes. The priority of the diagnosis body is to examine the collected materials and compare with patients' individual records; the records can save patients' baseline data, related doctors, treatment history, and other personal information. When abnormal subjects are detected, the diagnosis body can use external services, like text, to notify related doctors to take appropriate action.

The database's main function is to accept requests from the monitoring center staff, look for patients' medical records in the database, and send feedback data to the monitoring center and doctor client end. If the patient has no previous medical information, then new information can be established and saved to the database.

The monitoring client end consists of portable equipment, such as a mobile phone, PAD or tablet; it provides users with a method of software and hardware overall packaging and facilitates patients and families to question or interact. The each sensor node uses the nesC language to program the corresponding monitoring functions. The working process of wireless sensor nodes is shown in Figure 7.

\section{RESULTS}

After designing the wireless sensor nodes and relay nodes, the nodes need to be encapsulated. According to the system section, the packaging is also different. For example, the PVDF pulse sensor is encapsulated by wrist straps, so patients can it carry easily. For relay nodes, due to the high requirement of operability, communication ability and energy, the system is powered by lithium batteries and solar batteries, so a long-term and stable operation of the system can be ensured. Figure 8 shows the completed monitoring system.

To verify the measurement accuracy of this monitoring system, ten patients (5 male and 5 female, ages 18 69 with an average age of 41.8) were given a clinical test with this system. The monitoring center sent the mobile agent, which controls the wireless sensor node to collect the data on the physical condition of the patient, sent the results to the community monitoring center equipment after handling, and showed the date on an LCD display

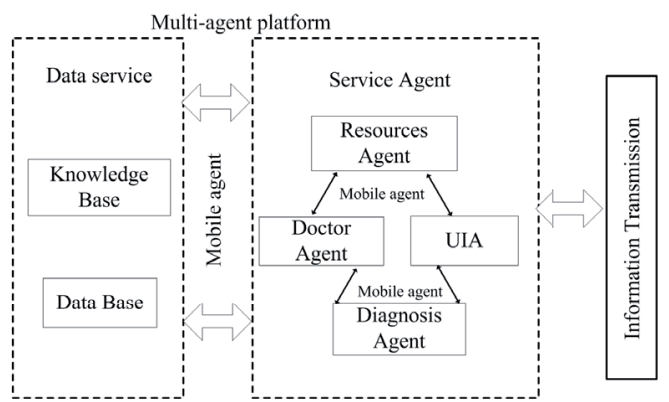

Figure 6. Mobile agent architecture

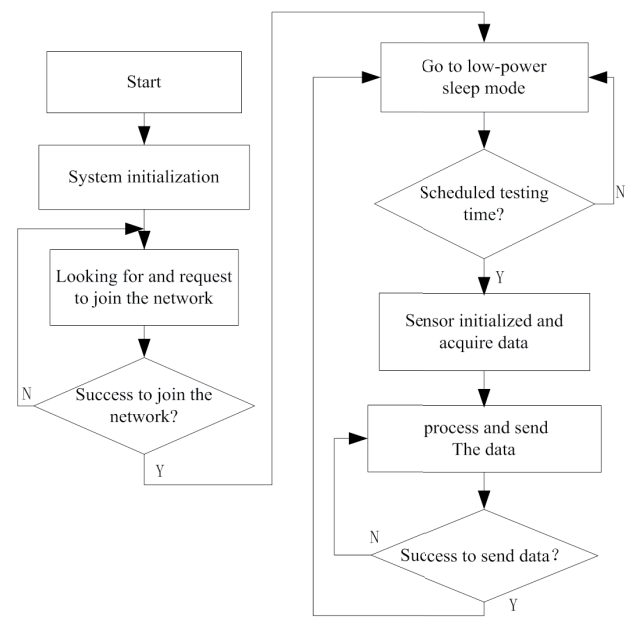

Figure 7. Structure diagram of sensor nodes

screen or PC. At the same time, the data could be sent to the hospital's monitoring center by an Internet of Things system as needed. The monitoring equipment dealt with the data received, removed the disturbances, and picked up the human health characteristic information, which can be saved in the database for searching later and can be also shown on PC and PDA equipment in the form of data and curvilinear figures. To ensure the reliability of the monitoring data, synchronous detection and comparison of the monitoring results obtained from this monitoring system and the Philips G80 multi parameter monitor were carried out. Table 1 shows the comparison results for the heart rate, blood pressure, degree of blood oxygen saturation, pulse, and body temperature, etc. Figures 9 (a) and (b) show the acquisition of the original ECG signal and the signal processed by the system, respectively. From Table 1 and Figure 9, it can be seen that the data from the monitoring system are stable and reliable, which can realize the collection and long-distance transmission of human health status information. A G80 multi parameter monitor is usually used in the hospital to monitor the vital signs parameters of the severe patients, but due to the large volume, expense, and inconvenience for patients, it is hard for the common patients to use without affecting daily living. This remote medical monitoring system is based on the Internet of Things and mobile agent technology and can give online monitoring of the heart rate, blood pressure, degree of blood oxygen saturation and pulse of the patients quickly and conveniently by using portable devices such as wireless sensor nodes, RFID tags, and so on. It can be installed and carried conveniently so the patients can adopt this monitoring system in daily life without affecting walking and work, showing its usefulness and significance. 
PAPER

Prototype Design of A Remote Medical Monitoring System Based on the InTERnet of Things

TABLE I.

CLINICAL TEST COMPARISON FOR MULTI-PARAMETERS

\begin{tabular}{|c|c|c|c|c|c|c|c|c|c|c|}
\hline \multirow{2}{*}{ Case } & \multicolumn{2}{|c|}{ heart rate } & \multicolumn{2}{|c|}{$\begin{array}{c}\text { blood pressure (systolic } \\
\text { pressure/diastolic pressure) }\end{array}$} & \multicolumn{2}{|c|}{ pulse } & \multicolumn{2}{|c|}{ body temperature } & \multicolumn{2}{|c|}{$\begin{array}{l}\text { degree of blood oxy- } \\
\text { gen saturation }\end{array}$} \\
\hline & $\begin{array}{c}\text { monitor- } \\
\text { ing system }\end{array}$ & $G 80$ & $\begin{array}{c}\text { monitoring } \\
\text { system }\end{array}$ & $G 80$ & $\begin{array}{c}\text { monitor- } \\
\text { ing system }\end{array}$ & $G 80$ & $\begin{array}{c}\text { monitor- } \\
\text { ing system }\end{array}$ & $G 80$ & $\begin{array}{c}\text { monitor- } \\
\text { ing system }\end{array}$ & G80 \\
\hline patient 1 & 64 & 65 & $126 / 82$ & $124 / 80$ & 65 & 65 & 36.8 & 36.8 & 97 & 96 \\
\hline patient 2 & 75 & 75 & $132 / 80$ & $130 / 81$ & 74 & 75 & 36.9 & 37.0 & 95 & 95 \\
\hline patient 3 & 66 & 67 & $120 / 78$ & $121 / 80$ & 66 & 66 & 36.5 & 36.6 & 96 & 96 \\
\hline patient 4 & 80 & 80 & $138 / 87$ & $139 / 85$ & 79 & 81 & 37.2 & 37.1 & 91 & 90 \\
\hline patient 5 & 59 & 60 & $116 / 78$ & $118 / 78$ & 60 & 60 & 36.4 & 36.5 & 95 & 94 \\
\hline patient 6 & 62 & 61 & $107 / 67$ & $109 / 66$ & 61 & 61 & 36.8 & 36.9 & 96 & 95 \\
\hline patient 7 & 89 & 87 & $142 / 90$ & $140 / 89$ & 88 & 88 & 37.5 & 37.6 & 92 & 92 \\
\hline Patient8 & 75 & 76 & $119 / 78$ & $120 / 81$ & 75 & 75 & 36.5 & 36.5 & 93 & 93 \\
\hline Patient9 & 78 & 78 & $112 / 75$ & $113 / 76$ & 77 & 78 & 36.6 & 36.6 & 95 & 95 \\
\hline Patient10 & 70 & 69 & $126 / 77$ & $120 / 76$ & 69 & 69 & 36.9 & 36.8 & 96 & 96 \\
\hline
\end{tabular}

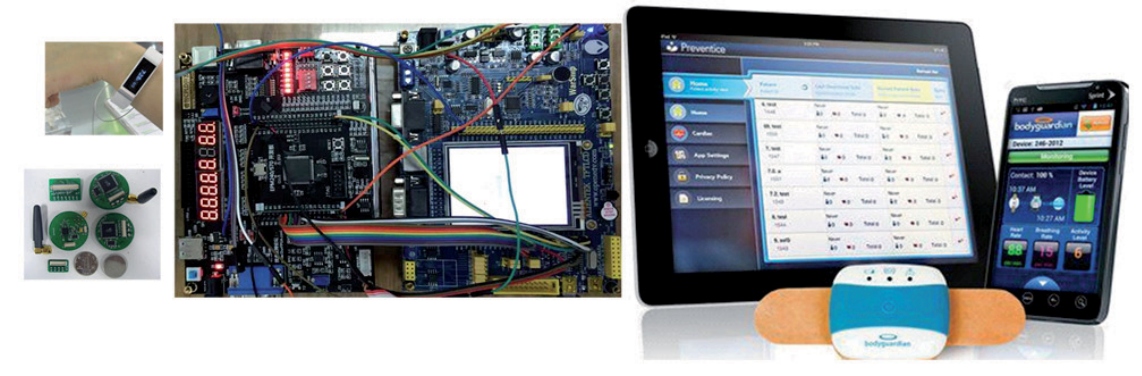

Figure 8. Physical monitoring system diagram

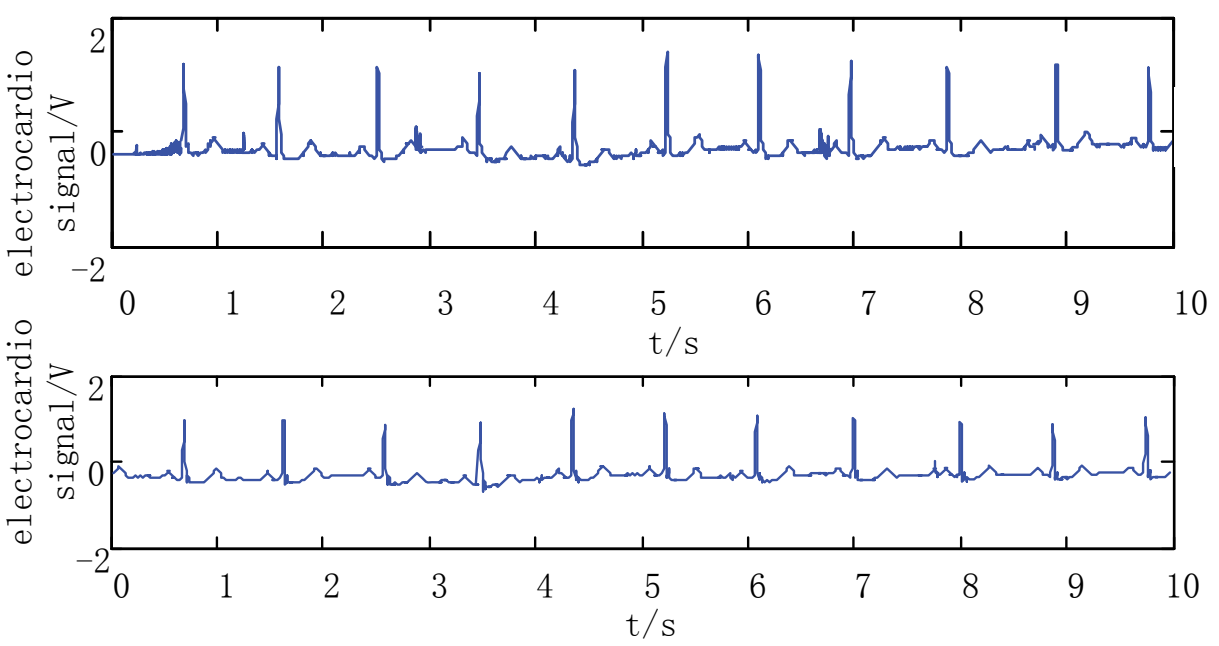

Figure 9. ECG signals of the former (a) and latter (b) filtering

\section{CONCLUSION}

This paper puts forward a remote medical monitoring system based on the Internet of Things and mobile agent, which can remotely monitor the health state of patients in real time. The system introduces a mobile subject and overcomes remote monitoring systems' dependence on network reliability and stability, large amounts of data communication, lack of real-time monitoring, and adaptability of the heterogeneous system. And at the same time, it greatly improves the system's scalability, adaptability, and intelligence and can meet the requirements for remote medical treatment. From the position of the medical staff, it can monitor patients' physiological parameters in real time and reduce the workload of medical staff. From the perspective of the patients, communication through wireless sensor nodes, RFID tags, and main subjects can quickly identify patients' information, effectively improve the medical care of patients, fully adapt to the distribution characteristics of healthcare environments, and realize optimized utilization of medical resources. The experimental results show that the effect of remote monitoring is good, and the system operates reliably. However, security problems with mobile body technology itself also need further improvement. 
PAPER

Prototype Design of a Remote Medical Monitoring System Based on the InTERnet of Things

\section{REFERENCES}

[1] E. Palm, A. Nordgren, M. Verweij, G. Collste. "Ethically sound technology? Guidelines for interactive ethical assessment of personal health monitoring," Interdisciplinary Assessment of Personal Health Monitoring, vol. 187, pp.105-114, 2013. http://dx.doi.org/10.3233/978-1-61499-256-1-105

[2] BD. Mittelstadt, BN. Fairweather, M. Shaw, N. Mcbride. "The ethical implications of personal health monitoring," International Journal of Technoethics, vol. 5, no. 2, pp.37-60, 2014. http://dx.doi.org/10.4018/ijt.2014070104

[3] Z. Pang, L. Zheng, J. Tian, S. Kao-Walter, EDQ. Chen, "Design of a terminal solution for integration of in-home health care devices and services towards the Internet-of-Things," Enterprise Information Systems, vol. 9, no. 1 pp. 86-116, 2013. http://dx.doi.org/10.1080/17517575.2013.776118

[4] M. Swan. "Sensor mania! The Internet of Things, wearable computing, objective metrics, and the quantified self 2.0," Journal of Sensor and Actuator Networks, vol. 1, no. 3, pp.217-253, 2012. http://dx.doi.org/10.3390/jsan1030217

[5] JA. Stankovic. "Research directions for the Internet of Things," Internet of Things Journal, IEEE, vol. 1, no. 1, pp. 3-9, 2014. http://dx.doi.org/10.1109/JIOT.2014.2312291

[6] Jose Luis Calvo Rolle, Jose Luis Casteleiro Roca, Xosé Manuel Vilar Martinez, Juan Aurelio Montero Sousa, "Expert system development to assist on the verification of "TACAN" system performance," DYNA, vol. 89, no. 1, pp. 112-121, 2014.

[7] JS. Bhatia, C. Singh. "Impact of usage of discrete networks on Telemedicine capabilities especially in India," International Conference on Medical Imaging, M-health \& Emerging Communication Systems (MedCom), pp. 311-318, 2014. http://dx.doi.org/10.1109/medcom.2014.7006024

[8] V. Vaidehi, M. Vardhini, H. Yogeshwaran, G. Inbasagar, R. Bhargavi, C. Sweetlin Hemalatha, "Agent based health monitoring of elderly people in indoor environments using wireless sensor networks," Procedia Computer Science, vol. 19, pp.64-71, 2013. http://dx.doi.org/10.1016/j.procs.2013.06.014

[9] S. Cai, "The design of the smart pre-dust of intelligent monitoring system," Journal of Xiamen University (Natural Science), vol. 53, no. 4, pp.607-610, 2014.

[10] C. Perera, A. Zaslavsky, P. Christen, D. Georgakopoulos, "Sensing as a service model for smart cities supported by Internet of Things," Transactions on Emerging Telecommunications Technologies, vol. 25, no. 1, pp. 81-93, 2014. http://dx.doi.org/10.1002/ett.2704
[11] D. Cullar, D. Estrin, M. Srivastava, "Guest editors' introduction: Overview of sensor networks," Computer, vol. 37, no. 8, pp.4149, 2004. http://dx.doi.org/10.1109/MC.2004.93

[12] D. Izadi, J. Abawajy, S. Ghanavati, "Fuzzy logic optimized wireless sensor network routing protocol," Journal of High Speed Networks, vol. 19, no. 2, pp.115-128. 2013.

[13] H. Sun, S. Yuan, X. Zhao, H. Zhou, D. Liang, "Technology of structure damage monitoring based on multi-Agent," Journal of Systems Engineering and Electronics, vol. 21, no. 4, pp. 616-622, 2010. http://dx.doi.org/10.3969/j.issn.1004-4132.2010.04.014

[14] CJ. Su, CY. Wu, "JADE implemented mobile multi-agent based, distributed information platform for pervasive health care monitoring," Applied Soft Computing, vol. 11, no. 1, pp.315-325, 2011 http://dx.doi.org/10.1016/j.asoc.2009.11.022

[15] H. Hu, F. Zhang, "A new clustering model for wireless sensor networks," Journal of Computer science and Technology, vol. 2, pp.211-214, 2006.

[16] HT. Dinh, L Chonho, N. Dusit, P Wang, "A survey of mobile cloud computing: architecture, applications, and approaches," Wireless communications and mobile computing, vol. 13, no. 18, pp.1587-1611, 2013. http://dx.doi.org/10.1002/wcm.1203

[17] Z. Wu, "Optimization of Distribution Route Selection Based on Particle Swarm Algorithm," International Journal of Simulation Modelling, vol. 13, no. 2, pp.230-242, 2014. http://dx.doi.org/10.2507/IJSIMM13(2)CO9

[18] X Luo, "An Algorithm of Wavelet Data Compression Based on Wireless Sensor Network," Journal of Digital Information Management, vol. 11, no. 1, pp54-61, 2013.

[19] V. Kanakaris, D. Ndzi, D. Azzi, "Ad-hoc Networks Energy Consumption: A review of the Ad-Hoc Routing Protocols," Journal of Engineering Science and Technology Review, vol. 3, no. 1, pp. 162-167, 2010.

\section{AUTHOR}

Xiuming ZOU is an associate professor at the School of Physics and Electronic Electrical Engineering, Huaiyin Normal University, Huaian, Jiangsu, 223300, China. His research interests include wireless sensor technology, pattern recognition, machine learning and bioinformatics. (E-mail: brightzou@126.com).

This work is supported by the Key University Science Research Project of Jiangsu Province, China (No. 15KJA460004). Submitted 08 November 2015. Published as resubmitted by the authors 23 December 2015. 